\title{
Suporte Familiar, Auto-Eficácia e Lócus de Controle Evidências de Validade entre os Construtos
}

Family support, self-efficacy and locus of control: validity evidence between constructs

Makilim Nunes Baptista, Gisele Aparecida da Silva Alves Universidade de São Francisco

Thelma Margarida de Moraes dos Santos Universidade Sagrado Coração (USC) - Bauru- SP
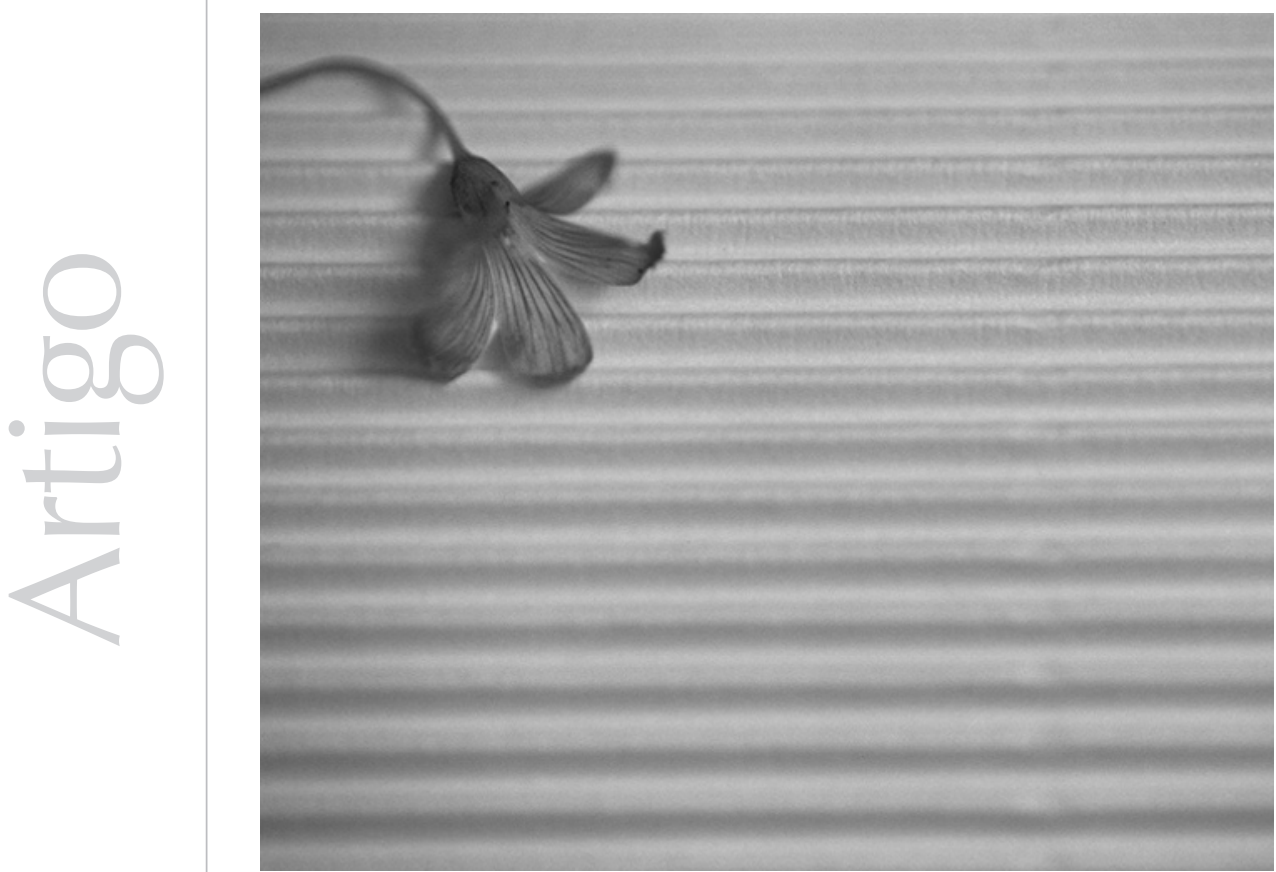
Resumo: O objetivo deste estudo foi correlacionar a percepção do suporte familiar com lócus de controle e auto-eficácia em 403 estudantes de uma universidade particular do Estado de São Paulo. Os instrumentos utilizados foram o inventário de percepção de suporte familiar (IPSF), a escala de lócus de controle de Levenson (ELCL) e a escala de auto-eficácia geral de Sherer (GSES). Todas as dimensões do IPSF (adaptação, autonomia e afetivo-consistente) se correlacionaram negativamente com a dimensão externa do lócus de controle. Ao correlacionar as dimensões do IPSF com a dimensão interna do lócus de controle, foram encontradas correlações positivas somente com a dimensão denominada afetivo-consistente. Já nas correlações das dimensões do IPSF com a GSES, todas as dimensões do IPSF se correlacionaram positivamente. Nesse sentido, observou-se associação entre percepção do suporte familiar, lócus de controle e auto-eficácia, demonstrando-se mais uma evidência de validade baseada na relação com outras variáveis para o IPSF.

Palavras-chave: Avaliação psicológica. Família. Auto-eficácia. Lócus de controle.

\begin{abstract}
The aim of this study was to correlate the perception of family support with locus of control and self-efficacy in 403 students who attended a private University located in the state of São Paulo. The instruments used in this study were the perception of family support inventory (IPSF), Levenson's locus of control scale (ELCL) and Sherer's general self-efficacy scale (GSES). All dimensions of IPSF were negatively correlated to the external dimension of locus of control. When correlating the IPSF dimensions with the internal dimension of locus of control, the authors found positive correlations with the dimension called affective-consistent. In the correlations between IPSF dimensions and GSES, all dimensions were positively correlated. So, associations among the perception of family support, locus of control and self-efficacy were observed, leading to one more validity evidence based on other variables.
\end{abstract}

Keywords: Psychological assessment. Family. Self-efficacy. Locus of control.

McFarlane,

Belíssimo e

Norman (1995)

definem suporte

familiar como

manifestações

de carinho,

atenção, diálogo,

proximidade

afetiva, liberdade

e independência

entre os membros da família,

podendo ser esse considerado

um construto multidimensional

e de complexa operacionalização, já que a literatura não oferece uma definição padrão de suporte familiar.
Na tentativa de entender o comportamento na área da saúde mental, pesquisas têm enfocado as influências de fatores psicológicos, biológicos, sociais e ambientais, no intuito de avaliar que variáveis estão relacionadas com o desenvolvimento e a manutenção de problemas psicológicos bem como de compreender porque os indivíduos adoecem. Para se entender a aquisição e a mudança de comportamento nessa área, a literatura disponibiliza alguns estudos acerca de variáveis que podem influenciar os cursos de ação dos indivíduos, tais como o suporte familiar, a auto-eficácia e o lócus de controle (Baptista, Santos, \& Dias, 2006; Holloway \& Watson, 2002; Regehr, Hill, Knorr, \& Saulr, 2003).
McFarlane, Belíssimo e Norman (1995) definem suporte familiar como manifestações de carinho, atenção, diálogo, proximidade afetiva, liberdade e independência entre os membros da família, podendo ser esse considerado um construto multidimensional e de complexa operacionalização, já que a literatura não oferece uma definição padrão de suporte familiar. Olson, Russell e Sprenkle (1983) se fundamentaram na teoria sistêmica familiar para apresentar um modelo de interação da família que consiste em três dimensões principais, denominadas coesão, adaptabilidade e comunicação, para a compreensão do funcionamento familiar. 
Atualmente existem poucas escalas desenvolvidas no Brasil e na América Latina que avaliam a percepção de suporte familiar com diversas evidências de validade (Baptista, 2005; Teodoro, 2006). Nesse sentido, o inventário de percepção de suporte familiar (IPSF), construído e desenvolvido no Brasil (Baptista, 2005; Baptista, 2007), é um instrumento que vem demonstrando boas qualidades psicométricas e já possui evidências de validade de construto, e baseada na relação com outras diversas variáveis.

A validade poderia ser definida como um procedimento que visa a verificar se determinado instrumento realmente mede o que se propõe a medir. Trata-se de um conceito único, portanto, não há tal aspecto comotipos diferentes de validade, e sim, diferentes tipos de evidência que podem iluminar diferentes aspectos da validade (American Educational Research Association, American Psychological Association, \& National Council on Measurement in Education [AERA, APA, \& NCME], 1999).

Uma das evidências de validade para o desenvolvimento de um instrumento com adequadas qualidades psicométricas seria a evidência de validade baseada na relação com outras variáveis. Sendo assim, propõese a importância da análise da relação dos escores de um teste com outras variáveis externas, que possam incluir as medidas de alguns critérios que o teste se propõe a medir, assim como as relações com outros testes que meçam construtos relacionados ou diferentes. Nesse sentido, as evidências encontradas entre esses construtos, por intermédio de instrumentos de medida confiáveis, indicariam o quanto essa relação é consistente com o construto destacado nas interpretações do teste (AERA, APA, \&
NCME, 1999), mesmo porque a eficácia do instrumental utilizado no processo de avaliação psicológica está diretamente ligada à quantidade e à qualidade de informações oriundas de pesquisas científicas acumuladas, relacionadas à interpretação dos resultados desses instrumentos (Primi, 2005).

Nesse sentido, alguns autores desenvolveram estudos que tiveram como objetivo buscar evidências de validade para o inventário de percepção do suporte familiar (Baptista, 2005), baseadas na relação com outras variáveis, que correlacionavam o suporte familiar com a vulnerabilidade ao estresse laboral (Aquino, 2007), traços de personalidade (Santos, 2005), suporte social e autoconceito (Rigotto, 2006), saúde mental (Souza, 2007) e desesperança (Baptista \& Alves, manuscrito submetido à publicação).

Além da relação do construto suporte familiar com variáveis como autoconceito, personalidade, vulnerabilidade ao estresse laboral e saúde mental, o suporte familiar, a auto-eficácia e o lócus de controle também parecem estar relacionados com outros construtos cognitivos e psicopatologias (Benassi, Sweeney, \& Dufour, 1988; Eisemann, Perris, Palm, \& Perris, 1988; Harrow \& Ferrante, 1969). Benight, Harding-Taylor, Midboe e Durham (2004) relatam que os modelos aprendidos no núcleo familiar se repetem nos relacionamentos interpessoais posteriores, o que pode explicar a relação entre a família e a maneira como os indivíduos desenvolvem modelos avaliativos em relação ao mundo e aos problemas do cotidiano. Algumas pesquisas relacionam o suporte familiar com a auto-eficácia e o lócus de controle (Kim \& Ciccetti, 2003; Malcarne, Dahota, \& Hamilton, 2005); no entanto, tais pesquisas ainda representam um número reduzido. 
Um indivíduo pode acreditar, por exemplo, que ser saudável (resultado) seja um determinante de seus próprios comportamentos, ou, de forma contrária, acreditar que a saúde não dependa, diretamente, de seus comportamentos, como, por exemplo, alimentação, exercícios físicos, dentre outros.
A auto-eficácia é um conceito utilizado pela teoria cognitivo social de Bandura (1986), podendo ser definida como a crença do indivíduo em sua capacidade de desempenho em atividades específicas, que envolvem o julgamento que as pessoas fazem do quanto são capazes de realizar e organizar atividades em situações diversas. Segundo Bandura (1977, 1982), a auto-eficácia pode ser considerada uma crença, ou seja, uma regra para a ação, e pode envolver aspectos cognitivos, emocionais e motores. Tais crenças podem afetar o desempenho das atividades escolhidas pelo indivíduo, o estabelecimento de metas e a perseverança perante as dificuldades que ele mesmo encontra durante sua execução (Bem, 1972).

De acordo com Rotter (1966), o comportamento dos indivíduos também varia em função de expectativas generalizadas de que os resultados de suas ações sejam determinados pelas próprias ações (lócus de controle interno) ou por fatores externos, que fogem ao controle do indivíduo (lócus de controle externo) (Bandura, 1986; Rotter, 1966). DeMoulin (1993) também relata, em um estudo de revisão teórico, que a eficácia pode ser constituída de dois componentes básicos: lócus de controle e expectativas de eficácia, mas salienta que a auto-eficácia também pode ser encontrada na literatura como independente do lócus de controle. Tendo em vista essa distinção, Holloway e Watson (2002), ao citar Bandura, relatam que este afirma que o indivíduo pode possuir uma orientação interna do lócus de controle, mas não ser auto-eficaz.

A principal razão para essa distinção é que o indivíduo pode acreditar que um resultado específico possa ser conseguido, mas que ele seja incapaz de fazê-lo. Um indivíduo pode acreditar, por exemplo, que ser saudável (resultado) seja um determinante de seus próprios comportamentos, ou, de forma contrária, acreditar que a saúde não dependa, diretamente, de seus comportamentos, como, por exemplo, alimentação, exercícios físicos, dentre outros. Sendo assim, salienta-se que, se os indivíduos acreditam que sejam incapazes de produzir um resultado específico, eles provavelmente não iniciarão a ação necessária para atingi-lo (Holloway \& Watson, 2002; Silva, 2004).

O construto lócus de controle pode ser definido como a expectativa generalizada de alguém em sua capacidade de controlar os acontecimentos (reforços) que se seguem às suas ações. Tal construto se reporta à teoria da aprendizagem social (Rotter, 1966). Rotter discute ainda a multidimensionalidade do lócus de controle. O mesmo possuiria três dimensões, uma interna e duas externas. A dimensão interna se relaciona à percepção do indivíduo sobre a conseqüência de seus comportamentos sob o controle de suas próprias ações, e a externa, se a conseqüência de seus comportamentos estiverem relacionadas a fatores externos, pode-se atribuir o controle a outros fatores poderosos, como à sorte ou ao destino. Sendo assim, algumas pesquisas que envolvem suporte familiar, auto-eficácia e lócus de controle serão descritas a seguir.

Hoeltje, Zubrick, Silburn e Garton (1996) avaliaram o suporte familiar e a autoeficácia em 260 crianças entre 12 e 16 anos, demonstrando que, quanto maior a quantidade de carinho recebido, interesse, acolhimento, comunicação adequada, habilidades em resolução de problemas, clareza de regras e consistência entre o verbal e o comportamental recebidos pela família, tanto maior a percepção que o indivíduo terá de se considerar capaz de 
realizar determinadas tarefas. Observase, então, a importância da família como local de socialização primária nas crenças desenvolvidas pelo indivíduo.

Mamlim, Harris e Case (2001), ao verificarem as relações que sexo, idade e cargo exercido estabelecem com o lócus de controle, relataram tendências para orientações específicas de lócus de controle, sendo as três tendências principais descritas a seguir: homens tendem a possuir uma orientação mais interna quando comparados às mulheres; conforme as pessoas ficam mais velhas, o lócus de controle tende a ser mais interno, e pessoas com cargos mais altos em organizações tendem a ter orientações mais internas.

Em um estudo com 159 idosos chineses com doenças crônicas, Wu, Tang e Kwok (2004) encontraram associação entre auto-eficácia e lócus de controle interno, em que a autoeficácia se correlacionava negativamente com a dimensão interna do lócus de controle e positivamente com a dimensão externa. Isso pode ser devido ao fato de a amostra ser composta por idosos portadores de doenças crônicas, em que não há nada efetivo que se possa fazer para a cura da doença. No entanto, estudos mais comumente encontrados na literatura existente na área têm mostrado associações positivas entre auto-eficácia e lócus de controle interno, como o de Kaplan, Atkins e Reinsch (1984), ao estudar pacientes idosos portadores de doença pulmonar.

Sendo assim, o objetivo principal desta pesquisa foi o de avaliar a evidência de validade baseada na relação com outras variáveis para o inventário de percepção de suporte familiar (IPSF), utilizando-se a escala de lócus de controle de Levenson (ELCL) e a escala de auto-eficácia geral (GSES). Como objetivos secundários, foram avaliados se sexo, estado civil, classe social e situação dos pais trazem diferenças para os escores de percepção do suporte famíliar, lócus de controle e auto-eficácia.

\section{Método}

\section{Participantes}

Participaram desta pesquisa 403 estudantes de graduação de uma universidade privada do interior do Estado de São Paulo, sendo que a idade variou de 17 a 64 anos e teve como média 23,33 e desvio padrão de 6,23 anos, sendo a maioria do sexo feminino $(80,2 \%)$. Em relação ao estado civil, observou-se que $89 \%$ dos participantes eram solteiros, e $11 \%$, casados. Já em relação à classe social, as porcentagens encontram-se a seguir: A1 $(2,7 \%), A 2(18,3 \%), B 1(24,3 \%), B 2(25,5 \%)$, C $(23,3 \%), D(5,7 \%)$ e $E(0,5 \%)$.

\section{Instrumentos}

- Questionário de identificação

Esse questionário foi desenvolvido pelos autores, a fim de colher dados sociodemográficos dos participantes. Anexado a ele, foi aplicado o questionário da ANEP Associação Nacional de Empresas de Pesquisa (ANEP, 2007), para a classificação das classes sociais. O questionário era composto de sete perguntas fechadas (estado civil, período em que estuda/trabalha atualmente, com quem mora atualmente, estado civil dos pais, classe social e nível de escolaridade dos pais) e três abertas (idade, curso e semestre).

- Inventário de percepção de suporte familiar (IPSF) (Baptista, 2005; Baptista, 2007)

O IPSF avalia a percepção que o indivíduo tem do suporte que recebe de sua própria família. 
É uma escala likert de 3 pontos, a saber, quase nunca ou nunca, as vezes e quase sempre ou sempre, e possui 42 itens. Em uma primeira análise fatorial (Baptista, 2005), o IPSF ficou constituído de 4 dimensões e 43 itens; no entanto, após a análise fatorial composta de 1064 participantes (Baptista, 2007), passou a conter três dimensões, denominadas a seguir com seus respectivos coeficientes alpha de Cronbach: afetivo-consistente $(0,91)$, que se refere à expressão de afetividade entre os membros familiares (verbal e não verbal), interesse, proximidade, acolhimento, comunicação, interação, respeito, empatia, clareza nas regras intrafamiliares, consistência de comportamentos e verbalizações e habilidades na resolução de problemas, adaptação familiar $(0,90)$, referente à ausência de sentimentos e comportamentos negativos em relação à família, tais como raiva, isolamento, incompreensão, relações agressivas (brigas e gritos), além de competitividade entre os familiares, e autonomia familiar $(0,78)$, referente a relações de confiança, liberdade e privacidade entre os membros. Todas as dimensões apresentaram eigenvalues superiores a 2,5, o que explica $41,4 \%$ da variância total, com cargas fatoriais acima de 0,30 .

- Escala de lócus de controle de Levenson (ELCL) (Dela Coleta, 1987)

A ELCL mede o quanto o indivíduo atribui acontecimentos de sua vida ao acaso, a outros fatores poderosos ou a si próprio, sendo constituído de 24 itens. É composto por uma escala likert de 5 pontos, a saber, "concordo totalmente", "concordo em parte", "indeciso", "discordo em parte" e "discordo totalmente". Possui duas dimensões, sendo uma interna, denominada " $\mathrm{I}$ ", e duas externas, denominadas " $\mathrm{P}$ " $\mathrm{e}$ " $\mathrm{C}$ ". A dimensão externa é subdividida em duas, a primeira chamada "acaso C" e a outra, "outros poderosos P". A confiabilidade da escala I, medida pelo método de Spearman-Brown, alcançou 0,54, ao mesmo tempo em que o coeficiente $\alpha$ de Cronbach alcançava 0,50. A escala "P" apresentou os valores 0,66 (Spearman-Brown) e 0,62 ( $\alpha$ de Cronbach). Finalmente, a escala " $C$ " apresentou valores 0,65 (SpearmanBrown) e 0,63 ( $\alpha$ de Cronbach).

- A escala de auto-eficácia geral (GSES) (Sherer \& Adams, 1983)

A GSES mede o quanto o indivíduo pensa ser eficiente para a realização de determinada tarefa. É composta por uma escala likert de 5 pontos, a saber, "discordo fortemente", "discordo moderadamente", "nem concordo nem discordo", "concordo moderadamente" e "concordo fortemente", e contém 30 itens. Embora haja três dimensões, uma delas, denominada filler, não compõe a pontuação total do instrumento, pois refere-se a perguntas não relacionadas ao construto. As outras duas são denominadas auto-eficácia geral e auto-eficácia social. As escalas foram testadas em 500 universitários e 150 internos de um hospital de reabilitação para alcoolistas, para os quais o alpha de Cronbach encontrado foi de 0,86 (Sherer \& Adams, 1983; Sherer et al., 1982). Os autores do presente trabalho realizaram a tradução dessa escala, enviando-a a um professor bilíngüe para que este traduzisse a escala para o português e repassando-a a outro professor bilíngüe, para que este passasse a escala novamente para o inglês. Após o envio da versão em inglês para o autor original da escala (Sherer), o mesmo propôs algumas pequenas modificações e consentiu que a escala fosse utilizada nesta pesquisa. 


\section{Procedimento}

Após o projeto ter sido encaminhado e aprovado pelo comitê de ética e termos solicitado a autorização dos coordenadores e docentes dos cursos em questão, os instrumentos foram aplicados de forma coletiva, em salas de aproximadamente 40 alunos cada. A seqüência da aplicação foi variável, a fim de evitar o viés da fadiga, de modo que o questionário de identificação era sempre o primeiro a ser respondido, seguido por seqüências variadas (ex: IPSF, ELCL, GSES ou ELCL, GSES, IPSF ou GSES, ELCL e IPSF). A aplicação levou em média 50 minutos, sendo que todos os instrumentos foram respondidos em uma única aplicação, após a assinatura do Termo de Consentimento Livre e Esclarecido.

\section{Análise dos resultados}

Os dados foram inseridos e tratados por intermédio do programa SPSS 11.5, utilizandose o nível de significância de 5\%. Foi utilizado o Teste de Pearson para avaliar as correlações entre o inventário de percepção de suporte familiar (IPSF), a escala de lócus de controle de Levenson (ELCL) e a escala de autoeficácia geral (GSES). Ao analisar os dados, os cursos foram divididos em áreas, a saber, exatas (Matemática, ciências da computação), humanas (Psicologia, Pedagogia, turismo), biológicas (nutrição, enfermagem, farmácia) e ciências sociais (relações públicas, Filosofia).

A seguir, encontra-se a tabela de correlações entre a percepção de suporte familiar com o lócus de controle e a auto-eficácia (Tabela 1).

Tabela 1. Correlações entre percepção de suporte familiar, lócus de controle e auto-eficácia.

\begin{tabular}{|c|c|c|c|c|c|}
\hline & \multicolumn{2}{|c|}{$\begin{array}{l}\text { GSES - Auto- } \\
\text { eficácia }\end{array}$} & \multicolumn{2}{|c|}{$\begin{array}{l}\text { ELCL - Lócus de } \\
\text { controle externo }\end{array}$} & \multirow[t]{2}{*}{$\begin{array}{l}\text { ELCL - Lócus de } \\
\text { controle interno }\end{array}$} \\
\hline & Geral & Social & Sorte/Azar & $\begin{array}{c}\text { Outros } \\
\text { poderosos }\end{array}$ & \\
\hline $\begin{array}{l}\text { IPSF - Afetivo } \\
\text { consistente }\end{array}$ & $0,25^{* *}$ & $0,13^{*}$ & $-0,11^{*}$ & $-0,20 * *$ & $0,15^{* *}$ \\
\hline IPSF - Adaptação & $0,21^{* *}$ & 0,02 & $-0,24^{* *}$ & $-0,20 * *$ & 0,0 \\
\hline IPSF - Autonomia & $0,11^{*}$ & 0,05 & $-0,1$ & $-0,15^{* *}$ & $0,10^{*}$ \\
\hline IPSF - Total & $0,25^{* *}$ & $0,10^{*}$ & $-0,16^{* *}$ & $-0,22 * *$ & $0,13^{*}$ \\
\hline
\end{tabular}

Como demonstrado na Tabela 1, todas as dimensões do IPSF (adaptação, autonomia e afetivoconsistente), bem como a pontuação total, se correlacionaram negativamente com a dimensão externa do lócus de controle, com nível de significância menor que 5\%, sendo que apenas na dimensão autonomia não houve correlação com a "sorte/azar" do lócus de controle externo. Ao correlacionar as dimensões do IPSF com a dimensão interna do lócus de controle, foram encontradas correlações positivas e estatisticamente significativas com as dimensões denominadas 
As análises dos dados sociodemográficos, utilizando-se o Teste $†$ de student, demonstraram que, em relação ao sexo, observouse que pessoas do sexo feminino tendem a possuir uma orientação mais externa do lócus de controle nas dimensões "outros poderosos" e "sorte/ azar", ou seja, acreditam que as conseqüências de suas ações sofrem mais influência de outras pessoas com maior

status, como, por exemplo, médico, professor, etc., ou ainda da própria sorte ou azar. afetivo-consistente, autonomia e total. Já nas correlações das dimensões do IPSF com a GSES, todas as dimensões do IPSF se correlacionaram positivamente com a dimensão auto-eficácia geral, ou seja, quanto maiores os escores de suporte familiar percebidos pelo indivíduo, maiores também os escores em auto-eficácia, e somente a totalidade do IPSF e a dimensão afetivoconsistente se correlacionaram positiva e significativamente com a dimensão auto-eficácia social, denotando que, quanto maiores os escores nas dimensões afetivo-consistente e total do IPSF, maiores também os escores em auto-eficácia social.

Tabela 2. Correlações entre o lócus de controle e a auto-eficácia.

\begin{tabular}{|c|c|c|c|c|}
\hline Variáveis & & ELCL - Lócus de cont & role externo & $\begin{array}{l}\text { ELCL - Lócus de } \\
\text { controle interno }\end{array}$ \\
\hline \multirow{3}{*}{ GSES - Auto-Eficácia } & & Outros poderosos & Sorte/Azar & \\
\hline & Geral & $-0,31^{* *}$ & $-0,17^{* *}$ & $0,29 * *$ \\
\hline & Social & $-0,14^{* *}$ & $-0,1$ & $0,15^{* *}$ \\
\hline
\end{tabular}

Nas correlações entre o lócus de controle (ELCL) e auto-eficácia (GSES), observou-se, na tabela 2, que as dimensões da GSES (geral e social) se correlacionaram positiva e significativamente com a dimensão interna do lócus de controle, ou seja, quanto maiores os escores de lócus de controle interno, maiores também os escores de auto-eficácia. Já nas correlações entre as dimensões do lócus de controle externo e as de auto-eficácia, observaram-se correlações negativas e significativas em todas elas, exceto entre a dimensão social de auto-eficácia e a dimensão sorte/azar do lócus de controle externo, ou seja, quanto maiores os escores de auto-eficácia, menores os escores no lócus de controle externo, exceto em sorte/azar, em que a correlação não foi significativa.

As análises dos dados sociodemográficos, utilizando-se o Teste t de student, demonstraram que, em relação ao sexo, observou-se que pessoas do sexo feminino tendem a possuir uma orientação mais externa do lócus de controle nas dimensões "outros poderosos" e "sorte/azar" "( $\mathrm{t}=0$, $846 ; p=0,04)$, ou seja, acreditam que as conseqüências de suas ações sofrem mais influência de outras pessoas com maior status, como, por exemplo, médico, professor, etc., ou ainda da própria sorte ou azar. Em relação à classe social, diferenças significativas foram encontradas nas dimensões do IPSF afetivo-consistente $(t=2,455 ; p<0,01)$ e no IPSF total $(t=2,075 ; p$ $<0,01$ ), demonstrando que pessoas que foram classificadas em nível maior percebem maior afetividade e consistência no suporte proveniente de suas famílias do que pessoas que foram classificadas em níveis menores. Já em relação ao estado civil dos participantes e dos pais, não foram encontradas diferenças estatisticamente significativas. Com relação à auto-eficácia, não houve diferenças de médias entre sexo, estado civil, classe social e situação dos pais e as dimensões da GSES denominadas auto-eficácia geral e auto-eficácia social.

\section{Discussão}

O objetivo principal desta pesquisa foi o de buscar evidências de validade baseada na relação com outras variáveis entre o inventário de percepção de suporte familiar com a escala de lócus de controle de Levenson e a escala de auto-eficácia geral. Os resultados demonstraram haver 
correlação entre os três instrumentos na grande maioria das dimensões, o que sugere que, apesar de não medirem os mesmos construtos, eles estão relacionados.

As correlações de todas as dimensões do IPSF, inclusive com a pontuação geral e a autoeficácia geral, demonstraram que, quanto maior a quantidade de carinho recebido, interesse, acolhimento, comunicação adequada, habilidades em resolução de problemas, clareza de regras e consistência entre o verbal e o comportamental recebidos pela família, tanto maior a percepção que o indivíduo terá para se considerar capaz de realizar determinadas tarefas. Segundo Hoeltje et al. (1996), a auto-eficácia contribui de forma importante para a sensação de bem-estar das pessoas e seu ajustamento perante situações adversas durante a vida; sendo assim, é de suma importância obter dados de como o indivíduo adquiriu tais habilidades, referindo-se à família como um dos primeiros ambientes (modelos) capazes de fornecer padrões de pensamento e comportamentos.

Ainda de acordo com Hoeltje et al. (1996), crianças e adolescentes que viveram em famílias equilibradas, em que havia um clima de respeito mútuo, com poucos conflitos e relacionamentos familiares funcionais, tendem a desenvolver a crença de que possuem habilidades em lidar efetivamente com mudanças adversas na vida, quando comparadas com aquelas crianças e adolescentes que cresceram em um clima familiar com baixo suporte. Dessa forma, Benight et al. (2004), ao avaliarem auto-eficácia em uma amostra de crianças maltratadas, encontraram baixas pontuações dessa medida, associadas à falta de otimismo, a estratégias de enfrentamento inadaptativas e ao funcionamento psicológico não-saudável. A auto-eficácia foi negativamente correlacionada com distress, humor negativo e estratégias de enfrentamento desadaptativas.

Kim e Ciccetti (2003), ao avaliarem uma amostra de 500 crianças entre 5 e 12 anos com grupos contrastantes (305 crianças maltradas e 195 crianças não maltratadas), relataram, em seu estudo, que crianças que perceberam o recebimento de carinho, atenção e afetividade por parte dos pais como deficitário também possuíam uma tendência de se julgarem menos capazes para desempenhar determinadas tarefas e de possuir déficits no desenvolvimento de autonomia, o que corrobora os dados encontrados neste estudo.

Da mesma forma, Oliver e Paull (1995), em um estudo com 186 estudantes universitários que avaliava relações entre auto-eficácia, auto-estima, suporte familiar e depressão, relataram haver encontrado correlações positivas entre a percepção que o indivíduo possui do suporte que recebe de sua família e o julgamento do indivíduo acerca da capacidade que possui para desempenhar tarefas em geral, ou seja, quanto maiores os escores de percepção de suporte familiar do indivíduo, também maiores os escores dos instrumentos que mediram a auto-eficácia.

Em relação ao lócus de controle e sexo, Malcarne et al. (2005) examinaram as diferenças de gênero e raça e o lócus de controle em 167 crianças estudantes de escolas públicas com idade entre 7 e 17 anos, e encontraram dados semelhantes aos resultados desta pesquisa. Malcarne et al. (2005) relatam que sujeitos do sexo feminino tendem a possuir um lócus de controle mais externalizado, ou seja, atribuem a outras pessoas e ao meio ambiente o controle sobre o que ocorre em suas vidas. Neste estudo, 
verificou-se que sujeitos do sexo feminino atribuíam o controle sobre o que ocorria em suas vidas a "outros poderosos" e à "sorte", corroborando então dados do estudo citado anteriormente. No entanto, pesquisas como a de Carvalho et al. (2001), ao avaliar a maturidade emocional, o lócus de controle e a ansiedade em 26 pré-adolescentes obesos, não relatam diferenças significativas de gênero em relação ao lócus de controle. Os autores tecem a hipótese de que isso pode ter ocorrido devido a limitações no tamanho da amostra utilizada no estudo.

Na relação percepção do suporte familiar e classe social, segundo Rigotto (2006), a pobreza pode não estar diretamente relacionada à variação de percepção de suporte familiar, o que é oposto aos resultados encontrados nesta pesquisa, demonstrando que indivíduos com maior poder aquisitivo perceberiam o suporte familiar recebido como mais adequado.

Wu et al. (2004), ao associarem auto-eficácia, lócus de controle e distress psicológico em 159 mulheres chinesas idosas, encontraram correlações negativas entre a auto-eficácia e o lócus de controle externo, e correlações positivas entre a auto-eficácia e o lócus de controle interno, ou seja, quanto menor o senso de auto-eficácia dos sujeitos, maior a orientação externa no lócus de controle, e quanto maior o senso de auto-eficácia, maior a internalidade do indivíduo no lócus de controle. Esses resultados também são semelhantes aos encontrados por esta pesquisa.

De forma geral, das 20 correlações possíveis entre o IPSF e as dimensões dos inventários de auto-eficácia e lócus de controle, 16 foram significativos, o que demonstra a associação entre as medidas bem como a evidência de validade do IPSF baseada na relação com essas variáveis que medem construtos relacionados. Quanto às relações entre suporte familiar e os outros dois construtos estudados nesta pesquisa, a saber, auto-eficácia e lócus de controle, os dados relatados nos resultados deste estudo demonstraram que, quanto mais alto o suporte familiar percebido, maior também a medida de auto-eficácia e lócus de controle interno, e quanto mais baixo o suporte familiar percebido, maior a medida de lócus de controle externo.

Quanto às relações entre auto-eficácia e lócus de controle, observou-se que, quanto maior a medida de lócus de controle interno, maior também a auto-eficácia, e quanto maior a medida de lócus de controle externo, menor a auto-eficácia. Quanto às analises secundárias, observaram-se diferenças estatisticamente significativas nas variáveis sexo e classe social, o que demonstra que mulheres tendem a possuir uma orientação mais externa no lócus de controle e que, quanto maior o nível econômico, mais alta é a medida de suporte familiar. 


\section{Makilim Nunes Baptista *}

Doutor - Programa de Pós-Graduação Stricto Sensu em Psicologia da Universidade São Francisco (USF) -Itatiba$\mathrm{SP}$; bolsista produtividade $\mathrm{CNPq}$

\section{Gisele Aparecida da Silva Alves}

Graduanda de Psicologia da Universidade São Francisco (USF) - Itatiba-SP; bolsista PROBAIC-USF do PIC (Programa de Iniciação Científica)

\section{Thelma Margarida de Moraes dos Santos}

Mestre pelo Programa de Pós-Graduação Stricto Sensu em Psicologia da Universidade São Francisco (USF) Itatiba-SP; docente da Universidade Sagrado Coração (USC) - Bauru/SP

* Endereço para correspondência:

Programa de Pós-Graduação Strictu-Sensu em Psicologia

Apoio Executivo às Comissões de Pós-Graduação

Av. Alexandre Rodrigues Barbosa, 45 - Centro Cep: 13251-900, Itatiba-SP

E-mail: makilim.baptista@saofrancisco.edu.br

Recebido 27/07/2007 Reformulado 07/11/2007 Aprovado 19/11/2007

American Educational Research Association, American Psychological Association, \& National Council on Measurement in Education. (1999). Standards for educational and psychological testing. New York: American Psychological Association.
Aquino, R. R. (2007). Inventário de percepção de suporte familiar (IPSF) e escala de vulnerabilidade ao estresse no trabalho (EVENT): evidência de validade. Dissertação de Mestrado em Psicologia, Programa de Pós-Graduação Stricto-Sensu em Psicologia, Universidade São Francisco, Itatiba, SP. 
Associação Nacional de Empresas de Pesquisa (ANEP). Critério de Classificação Econômica Brasil. Obtido em 12 de janeiro de 2007 do World Wide Web: http:// anep.org.br.

Bandura, A. (1977). Social learning theory. New Jersey: Prentice Hall.

Bandura, A. (1982). Self-efficacy mechanism in human behavior. American Psychologist, 37, 122-147.

Bandura, A. (1986). Social foundations of thought and action: A social cognitive theory. New Jersey: Prentice-Hall.

Baptista, M. N. (2005). Desenvolvimento do inventário de percepção de suporte familiar (IPSF): estudos psicométricos preliminares. Psico-USF, 10(1), 11-19.

Baptista, M. N. (2007). Inventário de percepção de suporte familiar (IPSF): estudo componencial em duas configurações. Psicologia: Ciência e Profissão, 27(3), 496-509.

Baptista, M. N., \& Alves, G.A.S. (manuscrito submetido à publicação). Relação entre suporte familiar e desesperança: evidências baseadas na relação com outras variáveis. Psicologia em Revista.

Baptista, M. N., Santos, K. M., \& Dias, R. R. (2006). Auto-eficácia, lócus de controle e depressão em mulheres com câncer de mama. Psicologia Argumento, 24(44), 27-36.

Bem, D. J. (1972). Atitudes, crenças e convicções. São Paulo: Herder.

Benassi, V. A., Sweeney, P. D., \& Dufour, C. L. (1988). Is there a relation between locus of control orientation and depression? Journal of Abnormal Psychology, 97(3), 357-367.

Benight, C. C., Harding-Taylor, A. S., Midboe, A. M., \& Durham, R. L. (2004). Development and psychometric validation of a domestic violence coping self-efficacy measure (DV-CSE). Journal of Traumatic Stress, 16(6), 505-509.

Carvalho, A. M. P., Netto, J. R. C., Bugliane, M. A. P., Borges, C. D., Mariano, F. N., Brancaleoni, A. P. L., \& Gorayeb, R. (2001). Maturidade emocional, lócus de controle e ansiedade em pré-adolescentes obesos. Paidéia, 11(20), 39-47.

Dela Coleta, M. F. (1987). Escala multidimensional de lócus de controle de Levenson. Arquivos Brasileiros de Psicologia, 39(2), 79-97.

Demoulin, D. F. (1993). Re-conceptualizing the self-efficacy paradigm: Analysis of an eight-year study. Education, 114(2), 168-199.

Eisemann, M., Perris, C., Palm, U., \& Perris, H. (1988). Locpresentation of a Sweedish instrument for assessing locus of control. In C. Perris \& M. Eisemann (Eds), Cognitive psychotherapy: An update (pp. 88-111). Umea: Department of Psychiatry, Umea University.

Harrow, M., \& Ferrante, A. (1969). Locus of control in psychiatric patients. Journal of Consulting and Clinical Psychology, 35(5), 582-589.

Hoeltje, C. O., Zubrick, S. R., Silburn, S. R., \& Garton, A. F. (1996). Generalized self-efficacy: Family and adjustments correlates. Journal of Clinical Child Psychology, 25(4), 446453.

Holloway, A., \& Watson, H. E. (2002). Role of self-efficacy and behaviour change. International Journal of Nursing Practice, 8, 106-115.

Kaplan, R. M., Atkins, C. J., \& Reinsch, S. (1984). Specific efficacy expectations mediate exercise compliance in patients with copd. Health Psychology, 3, 223-239.
Kim, J., \& Ciccetti, D. (2003). Social self-efficacy and behaviour problems in maltreated and nonmaltreated children. Journal of Clinical Child and Adolescent Psychology, 32(1), 106-117.

MacFarlane, A. H., Belíssimo, A., \& Norman, G. R. (1995). The role of family and peers in social self efficacy: Links to depression in adolescence. American Journal of Orthopsychiatry, 65, 402-410.

Malcarne,V. L., Dahota, A. D., \& Hamilton, N. A. (2005). Children's health-related locus of control beliefs: Ethnicity, gender and family income. Children's Health Care, 34(1), 47-59.

Mamlin, N., Harris, K. R., \& Case, L. P. (2001). A methodological analysis of research on locus of control and learning disabilities: Rethinking a common assumption. Journal of Special Education, 34(4), 214-225.

Oliver, J. M., \& Paull, J. C. (1995). Self-esteem and self-efficacy: Perceived parenting and family climate and depression in university students. Journal of Clinical Psychology, 51(4), 467-481.

Olson, D. H., Russel, C. S., \& Sprenkle, D. H. (1983). Circumplex model of marital and family systems: Theoretical update. Family Process, 22, 69-83.

Primi, R. (2005). Temas em avaliação psicológica. Campinas, SP: IDB Digital/Instituto Brasileiro de Avaliação Psicológica.

Regehr, C., Hill, J., Knorr, T., \& Saulr, B. (2003). Social support, self-efficacy and trauma in new recruits and experienced firefighters. Stress and Health, 19, 189-193.

Riggotto, D. M. (2006). Evidências de validade entre suporte familiar, suporte social e autoconceito. Dissertação de Mestrado em Psicologia, Programa de Pós-Graduação Stricto-Sensu em Psicologia, Universidade São Francisco, Itatiba, SP.

Rotter, J. (1966). Generalized expectancies for internal versus external control of reinforcements. Psychological Monographs, 80(609), 75-93.

Santos, T. M. M. (2005). Evidência de validade entre percepção de suporte familiar e traços de personalidade. Dissertação de Mestrado em Psicologia, Programa de Pós-Graduação Stricto Sensu em Psicologia, Universidade São Francisco, Itatiba, SP.

Sherer, M., \& Adams, C. H. (1983). Construct validation on the self-efficacy scale. Psychological Reports, 53, 899-902.

Sherer, M., Maddux, J. E., Marcadante, B., Prentice-Dunn, S., Jacobs, B., \& Rogers, R. W. (1982). The self-eficacy scale: Construction and validation. Psychological Reports, 51, 663-671.

Silva, A. M. M. (2004). Teoria da auto-eficácia. In M. F. Dela Coleta (Org.), Modelos para pesquisa e modificação de comportamentos em saúde (pp. 26-40). Taubaté, SP: Cabral Editora; Livraria Universitária.

Souza, M. S. (2007). Suporte familiar e saúde mental: evidência de validade baseada na relação com outras variáveis. Dissertação de Mestrado em Psicologia, Programa de Pós-Graduação Stricto-Sensu em Psicologia, Universidade São Francisco, Itatiba, SP.

Teodoro, M. L. M. (2006). Afetividade e conflito em díades familiares: avaliação com o familiagrama. Interamerican Journal of Psychology, 40, 385-390.

Wu, A. M. S., Tang, C. S., \& Kwok, T. C. Y. (2004). Self-efficacy, health locus of control, and psychological distress in elderly Chinese women with chronic illnesses. Aging and Mental Health, 8(1), 21-28. 